\title{
Assessment of Rationality in Prescribing Practice of Antibiotics in Out Patient Department at Moradabad
}

\author{
Aditya Sharma $^{1 *}$, Neelkant Prasad ${ }^{2}$, Mayur Porwal ${ }^{2}$, Alankar Shrivastav ${ }^{1}$, Najam Ali Khan ${ }^{1}$ \\ ${ }^{1}$ School of Pharmaceutical Sciences, IFTM University, Moradabad, Uttar Pradesh, INDIA. \\ ${ }^{2}$ Pharmacy Academy, IFTM University, Moradabad, Uttar Pradesh, INDIA.
}

\begin{abstract}
Objective: Rational use of drugs requires that patients receive medications appropriate to their clinical need. Prescription errors are the most common leading problems for patients and additional costs of the health system. Methodology: A prospective study was carried out after collecting 243 prescriptions of outpatients in the Moradabad city for a period of 3 months for evaluating the prescribing errors. Prescription's copies were obtained from the various community pharmacies of Moradabad. Result: The collected data was screened that such errors were almost in a similar proportion of the male patients as well as female patients $(54.08 \%$ and $51 \%$ respectively). Unauthorized synergistic combinations of antibiotics with drug-drug interactions were greater in male patients $(20 \%)$ than in female patients (7.69\%). Maximum error was found in advising and patient counselling i.e.77.14\% in male and $80.77 \%$ in female. Conclusion: Information on prescriptions and their incomplete or poor diagnosis may contribute to prescribing errors. Prescription errors need to be acknowledged, taken seriously, discussed openly and actions taken in a blame free culture.
\end{abstract}

Key words: Prescription, Antibiotics, Outpatient department (OPD), Error.

\section{INTRODUCTION}

Rational use of drugs is based on use of right drug, right dosage at right cost which is well reflected in the world health organization (WHO) definition: "Rational use of drugs requires that patients receive medications appropriate to their clinical needs, in doses that meet their own individual requirements for an adequate period of time, at the lowest cost to them and their community. ${ }^{1}$ Antimicrobial drug resistance refers to non-responsiveness of micro-organisms to an antimicrobial agent. It can be intrinsic or acquired. Acquired resistance develops due to widespread and irrational use of antimicrobials. Antimicrobial drug resistance is a growing problem and developing new antimicrobials is not the solution for this problem. ${ }^{2}$ It is the responsibility of the doctors to develop a good prescribing habit which will help in reducing the intensity of the problem. Some of the common causes that contribute to the development of antimicrobial resistance are an unnecessary use of antimicrobial drugs, inappropriate dose, inadequate duration of therapy, the use of irrational antimicrobial fixed dose drug combinations (FDCs). ${ }^{3}$ Because of the high level of community antimicrobial drug resistance the use of expensive drugs becomes mandatory which may not be affordable by most patients in developing countries like India. Another problem is many of the second and third line agents are becoming ineffective in clinical practice. And as it is reported earlier, 'the slow pace with which new molecules of antimicrobials are introduced into the market is inadequate to meet the needs of this global threat'. ${ }^{4}$ To tackle with this problem, global initiatives are trying to promote "antibiotic stewardship," with the aim of enhancing the appropriateness of antimicrobial use. But it requires continuous education of prescribers and patients, which needs to be supported
DOI: 10.5530/ijopp.10.4.53

Address for correspondence: Aditya Sharma, School of Pharmaceutical sciences, IFTM University, Moradabad-244001, Uttar Pradesh, INDIA.

Phone no: +91 8923371669 Email Id: adi99463@gmail.com

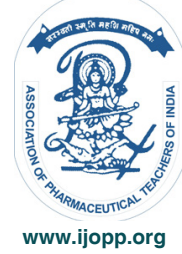


by high quality evidence linking antimicrobial use to the emergence of resistance. ${ }^{5}$ In view of all these issues associated with drug prescribing, the present study was conducted to delineate the prevailing prescribing practices and to know the extent of rational therapy in outpatient setup. A landmark report released by the Institute of Medicine in November 1999 stated that 44,000-98,000 people die each year in part because of medical errors over 7000 of these deaths attributed to medication errors. ${ }^{6}$ Various factors identified for medication errors are illegible orders, non- availability of patient information, inadequate medical knowledge, increased patient load, failure to monitor the drug concentration or drug therapy, and not accounting for changes in renal and cardiac functions. ${ }^{7}$

Currently, most Antimicrobial agents (AMAs) is employed in daily practice to treat infections as well as other diseases. The most serious misconsequence of this practice is the development of resistance in the population. A study has shown that a prescription of antibiotics taken with not more than five days doubles the chances of patients carrying antibiotic resistant bacteria. The same effect was not seen in patients who had taken antibiotics at least for the period of five days. ${ }^{8}$ Based on the above definitions, we can now clearly define the prescription error. The errors arising in the treatment of a patient are a medication error. It may arise at any stage of treatment like diagnosis, prescribing, dispensing or administration of the drug by the patient. If the error occurs at prescription stage, this may be referred to as prescription error. A clinically meaningful prescribing error occurs when because of a prescribing decision or prescription writing process, there is an unintentional significant reduction in the probability of treatment being timely and effective or increase in the risk of harm when compared with generally accepted practice. ${ }^{9}$ Such an error can be prevented from giving an adverse drug event by an interception of a person involved in the system. Hence the adverse drug events arising from prescription error can be classified as preventable adverse drug event. ${ }^{10}$ In terms of the components of the prescribing process, most of the errors concerned with the selection of the drug dose. ${ }^{11,12,13,14}$ The present study was conducted to evaluate the prescription errors related to the Drug-Drug Interactions, dosing, improper diagnosis, incomplete advice and patient counseling in Moradabad.

\section{MATERIAL AND METHODS}

This prescription's study was carried out after collecting 243 prescriptions of outpatients at a many chemist shop in Moradabad city. Prescriptions from newly registered patients were included in this study. After consultation with the physician, the prescriptions were copied, and the following index was determined.

\section{Period of Study}

This study was carried out in three months from Feb-April, 2016.

\section{Selection of Patient}

All the outpatients who were prescribed with antibiotic drugs were chosen for this study.

\section{Procedure for study}

The intended work was divided into the five steps

Step 1: Randomly arrangement of the prescriptions.

Step 2: To separate the prescriptions with antibiotics and without antibiotics.

Step 3: The prescriptions were divided into different age groups.

Step 4: Find out the errors regarding the use of antibiotics in the following

Aspects:

- Combinations of drug with the antibiotics, which interact to the Pharmacokinetics of antibiotics

- Improper synergistic combinations of antibiotics.

- Dose of antibiotics.

- Combinations of drug with antibiotics, which may cause serious Adverse Drug Reaction (ADR).

Step 5: To separate and count the number of prescriptions with each type of error in the different age groups represents them statistically and to find out the total number of prescriptions with errors related to antibiotics.

\section{RESULTS}

243 prescriptions have been reviewed, in which $60.49 \%$ $(n=147)$ were male and $(39.51 \%)(n=96)$ were female among these patients the highest number was in the adults and geriatrics (74.07\%) and lowest in child (6.17\%). The demographic data are shown in Table 1.

243 prescriptions were containing one or more antibiotics as the prescribed item of which $1.23 \%$ were containing 


\begin{tabular}{|c|c|c|c|c|}
\hline SI. No & Age group & Males & Females & Total (group wise) \\
\hline 1 & $\begin{array}{l}\text { Neonates and infants } \\
\text { (1month to } 2 \text { years) }\end{array}$ & $12(4.93 \%)$ & $12(4.93 \%)$ & $24(9.87 \%)$ \\
\hline 2 & $\begin{array}{c}\text { Child } \\
\text { (2 years to } 12 \text { years) }\end{array}$ & $06(2.46 \%)$ & $09(3.70 \%)$ & $15(6.17 \%)$ \\
\hline 3 & $\begin{array}{c}\text { Adolescent } \\
\text { (12 years to } 18 \text { years) }\end{array}$ & $18(7.40 \%)$ & $06(2.46 \%)$ & $24(9.87 \%)$ \\
\hline 4 & $\begin{array}{l}\text { Adults and geriatrics } \\
\text { (Above } 18 \text { years) }\end{array}$ & $111(45.67 \%)$ & $69(28.39 \%)$ & $180(74.07 \%)$ \\
\hline 5 & Total (sex wise) & $n=147$ & $\mathrm{n}=96$ & 243 \\
\hline
\end{tabular}

\begin{tabular}{|c|c|c|c|c|}
\hline SI. No & Route & Males & Females & Total (route wise) \\
\hline 1 & Topical & 00 & $1.23 \%$ & $1.23 \%$ \\
\hline 2 & Oral & $64.20 \%$ & $35.80 \%$ & $100 \%$ \\
\hline 3 & Parenteral & $7.40 \%$ & $1.23 \%$ & $8.64 \%$ \\
\hline
\end{tabular}

\begin{tabular}{|c|c|c|c|c|}
\hline & Age groups & Males & Females & Total (group wise) \\
\hline 1 & Neonates and infants & $06(50 \%)$ & $09(75 \%)$ & $15(62.5 \%)$ \\
\hline 2 & Children & $03(50 \%)$ & 00 & $03(20 \%)$ \\
\hline 3 & Adolescents & $09(50 \%)$ & $03(50 \%)$ & $12(50 \%)$ \\
\hline 4 & Adults and geriatrics & $69(62.16 \%)$ & $54(78.26 \%)$ & $123(68.33 \%)$ \\
\hline 5 & Total (sex wise) & $87(59.18 \%)$ & $66(68.75 \%)$ & $153(62.96 \%)$ \\
\hline
\end{tabular}

\begin{tabular}{ccccc}
\multicolumn{4}{l}{ Table 4: Percentage prescription errors of antibiotics administered in combination } \\
with any other drug. & Male & Female & Total \\
\hline SI. No & Error & Male & $06(7.69 \%)$ & $27(14.75 \%)$ \\
$\mathbf{1}$ & Drug-Drug Interactions & $21(20 \%)$ & $09(11.53 \%)$ & $12(06.56 \%)$ \\
$\mathbf{2}$ & Dose Related Error & $03(2.86 \%)$ & $144(78.69 \%)$ \\
3 & Advise and Patient & $81(77.14 \%)$ & $63(80.77 \%)$ & \\
\hline
\end{tabular}

topical preparations of antibiotics. Female predominance $(1.23 \%)$ was observed over male patients $(0 \%)$. Parenteral routes were used with a lower frequency $(8.64 \%)$ with $7.40 \%$ in male patients and $1.23 \%$ of female patients. Orally administered antibiotics were found to be prescribed with a very high frequency of $100 \%$. (64.20\% in male patients and $35.80 \%$ in female patients). The results are shown in Table 2.

Of the 243 prescriptions containing antibiotics, an overall error rate was found to be $62.96 \%$ of which $59.18 \%$ was related to male patients and $68.75 \%$ in female patients. The error rates were found to differ significantly in different age groups (62.5\% in neonates and infants , 20\% in children, $68.33 \%$ in adults and geriatrics and 50\% in adolescents); this is presented in Table 3 .
Errors related to the combination of antibiotics to the drugs affecting the pharmacokinetics of the given antibiotics were found to be $0 \%$ and the drug-drug interactions in any other way which may give rise to serious adverse drug reaction was found to be $14.75 \%$. Dose related error was found to be $06.56 \%$ and the error related to advising and patient counselling was found to be $78.69 \%$. Some of the important errors are summarized in Table 4.

Of the prescriptions neglecting the nutritional supplements, it was found that the adolescent age group was receiving $62.5 \%$ prescriptions having such an error and this was followed by children's group $(60 \%)$, adult and geriatrics $(55 \%)$ and neonates and infants $(75 \%)$

Indian Journal of Pharmacy Practice, Vol 10, Issue 4, Oct-Dec, 2017 


\begin{tabular}{|c|c|c|c|c|}
\hline SI. No & Age groups & Males & Females & $\begin{array}{c}\text { Total } \\
\text { (group wise) }\end{array}$ \\
\hline 1 & Neonates and infants & $09(75 \%)$ & $09(75 \%)$ & $18(75 \%)$ \\
\hline 2 & Children & $03(50 \%)$ & $06(66.66 \%)$ & $09(60 \%)$ \\
\hline 3 & Adolescents & $12(66.66 \%)$ & $03(50 \%)$ & $15(62.5 \%)$ \\
\hline 4 & Adults and geriatrics & $60(54.05 \%)$ & $39(56.52 \%)$ & $99(55 \%)$ \\
\hline 5 & Total (sex wise) & $84(57.14 \%)$ & $57(59.37 \%)$ & $141(58.75 \%)$ \\
\hline
\end{tabular}

The data showed that such errors were almost in a similar proportion of the male patients and female patients (57.14\% and $59.37 \%$ respectively). The outcome of management of nutritional supplements is given in Table 5.

\section{DISCUSSION}

The Current study provides the important information on prescribing pattern of antibiotic use in Moradabad district. This analysis report is precise and with true aspects. The prescription errors are related to the Drug-Drug Interactions, dosing, improper diagnosis, incomplete advice and patient counseling. It was seen that only one antibiotic prescribed for most patients, which was also reported by Mazzeo et al ${ }^{15}$ in a study carried out in an Italian university hospital. It was also found that some of those prescriptions had two or three antibiotics.

Prescribing error is the most serious error. Once an error has been made, unless detected, it will be systematically applied and can result in significant harm or death. In United Kingdom hospitals, prescribers make errors in $1.5 \%$ of prescriptions. ${ }^{16}$

Of all prescribed antibiotics, 8.64\% were injectable antibiotics. These injectable prescription patterns are lower than those found in studies conducted in Palestine $(62 \%)^{17}$ and Kathmandu Valley of Nepal $(75 \%) .{ }^{18}$ The WHO recommends only $10 \%$ of injectables for the total prescriptions for a health setting. ${ }^{19}$ The study has the following several limitations. There were significant $(23 \%)$ incomplete patient charts. The small sample size, the nature of the retrospective study may limit the generalizability of the findings. The public hospital-based nature of the study may also not be generalized to public health Centre's and private health institutions due to the different mix of health professionals and availability of drugs. It has been reported that distribution of highly skilled health professionals is skewed towards private and nongovernmental organizations in the Country. ${ }^{20}$ For example, in $2006-07,56 \%$ of specialists and $38 \%$ of general practitioners working in health facilities were outside the public sectors. ${ }^{21}$

In this analysis total 243 prescriptions were analyzed during the study period. Table No.1 shows the demographic characteristics of patients. Sexwise and age group wise distributions of patients were taking antibiotics during outpatient department (OPD). The number of female patients was comparatively less than the number of male patients. Patients were subdivided into four age groups as neonates and infants, child, adolescent and adults and geriatrics. Table No.2 showed the preferred routes of administration of Antibiotics in the prescriptions. It was found that in 243 prescriptions oral route was preferred a higher proportion of the drug prescribed between oral and parenteral routes of antibiotics during OPD. Table No.3 showed Percentage prescription errors in different age group patients. Table No.4 showed dose related error, drug-drug interactions error and advice and patient counselling related error was found to be maximum in the prescriptions. Table No.5 shows the percentage of prescriptions prescribing systemic Antibiotics without any nutritional supplement $58.75 \%$ prescriptions were prescribed without any nutritional supplement all prescriptions contain both generic and brand antibiotics.

\section{CONCLUSION}

In the study, the Information on prescriptions and their incomplete or poor diagnosis may contribute to prescribing errors. Prescription errors need to be acknowledged, taken seriously, discussed openly and actions taken in a blame free culture. There is an important need to develop a well-validated measure to assess the severity of prescribing errors that will better address their clinical significance and risk and collusion of pharmacist's in the current low profile of prescribing mistakes, giving verbal feedback to the prescriber, or writing on sticky notes must be stopped.

\section{ACKNOWLEDGEMENT}

The authors are grateful to the Hon'ble Vice Chancellor, 
IFTM University for his constant encouragement, valuable insight and facilities at all stages of this work. We specially acknowledge the Community pharmacies of Moradabad for provision of the data.

\section{CONFLICT OF INTEREST}

The authors declare no conflict of interest.

\section{ABBREVIATIONS USED}

OPD: Outpatient department; WHO: World health organization; AMAs: Antimicrobial agents.

\section{SUMMARY}

The current narrative study has demonstrated a high rate of prescribing errors of antibiotics use in Moradabad. In our study common prescribing errors are, regardless of the methods used for data collection or the medium used to order the medication. This directly indicates a clear need to reduce prescriptions errors, a conclusion supported by the findings of other large studies. Prescription errors may result in significant patient harm with potential clinical and legal consequences with wider implications for the National Health Service. All prescriptions required intervention by a clinical pharmacist before the drug could be dispensed in order to achieve optimal therapeutic outcomes for the patient.

\section{AUTHORS' CONTRIBUTION}

All authors have significantly contributed to this study with respect to research idea; design of the study; collection, analyses, and interpretation of data; and writing and submitting the manuscript.

\section{REFERENCES}

1. WHO. Model list of essential drugs, Geneva: World Health Organization. 1988.
2. Bhatia R, Narain JP. The growing challenge of antimicrobial resistance in the South-East Asia Region - Are we losing the battle? Indian J Med Res. 2010;132(5):482-6.

3. Ernest JS. Resistance to antimicrobials in humans and animals. BMJ. 2005;331(7527):1219-20.

4. Kumari IKS, Chandy SJ, Jeyaseelan L, Kumar R, Suresh S. Antimicrobial prescription patterns for common acute infections in some rural and urban Health facilities of India. Indian J Med Res. 2008;128(2):165-71.

5. Costelloe C, Metcalfe C, Lovering A, Mant D, Hay AD. Effect of antibiotic prescribing in primary care on antimicrobial resistance in individual Patients: systematic review and meta-analysis. BMJ. 2010;340:c2096.

6. Kohn LT, Corrigan JM, Donaldson MS, editors; Committee on Quality of Healthcare in America Institute of Medicine. To Err Is Human: Building a Safer Health System. Washington, DC: National Academy Press; 1999[online]. [Cited 2007 Aug16]. Available from URL: http://www.nap.edu/catalog/9728.html.

7. ASHP Council on Professional Affairs. American Society of Hospital Pharmacists. ASHP guidelines on preventing medication errors in hospitals. Am J Hosp Pharm. 1993;50:305-14.

8. Berwick DM. A primer on leading the improvement of systems. BMJ. 1996;312(7031):619.

9. Ghaleb MA, Barber N, Franklin BD, Wong CK. What constitutes a prescribing error in paediatrics. BMJ Quality and Safety in Health Care. 2005;14(5):352-7.

10. Mainous AG, Hueston WJ, Clark JR. Antibiotics and upper respiratory infection: Do some folks think there is a cure for the common cold? J Fam Pract. 1996;102:56-8.

11. Bates DW, Cullen DJ, Laird N. Incidence of adverse drug events and potential adverse drug events. Implications for prevention. ADE Prevention Study Group. JAMA. 1995;274(1):29-34.

12. Lesar TS, Briceland LL, Delcoure K. Medication prescribing errors in a teaching hospital. JAMA. 1990;263:2329-34.

13. Blum KV, Abel SR, Urbanski CJ. Medication error prevention by pharmacists. Am J HospPharm. 1988;45(9):1902-3.

14. Folli HL, Poole RL, Benitz WE. Medication error prevention by clinical pharmacists in two children's hospitals. Pediatrics. 1987;79(5):718-22.

15. Mazzeo F, Capuano A, Avolio A, Filipelli A, Rossi F. Hospital-based intensive monitoring of anti-bioticinduced adverse events in a university hospital. Pharmacol Res. 2004;51(3):269-74.

16. Dean M, Schachter VN. Research Pharmacist, Department of Practice and Policy, The School of Pharmacy, University of London, London WC1N 1AX, UK.

17. Ansam S, Ghada A, Laila A, Waleed S, Rowa A, Nidal J. Pattern of parenteral antimicrobial prescriptionamong paediatric patients in Al-Watani Government Hospital in Palestina. An-Najah Univ J Res. 2006;20:191-206.

18. Palikhe N. Prescribing pattern of antibiotics in paediatric hospital of Kathmandu valley. Kathmandu Univ Med J (KUMJ). 2004;2:6-12.

19. Isah A, Ross-Degnan D, Quick J, Laing R, Mabadeje A. The development of standard values forthe $\mathrm{WHO}$ drug use prescribing indicators. University of Benin, Nigeria; WHO. 2004.

20. AHO, WHO African Health Observatory and WHO: Analytical summary- Health workforce: Ethiopia. WHO. 2016.

21. FDRE Health Sector Development Program IV 2010/11 $\pm 2014 / 15$ Addis Ababa: Ministry of Health. 2010. 